\title{
BANACH-DIEUDONNÉ THEOREM REVISITED
}

\section{MONTSERRAT BRUGUERA and ELENA MARTÍN-PEINADOR}

\author{
Dedicated to Professor J. Arregui on the occasion of his 70th birthday
}

(Received 28 November 2000; revised 2 October 2002)

Communicated by G. Willis

\begin{abstract}
We prove that in the character group of an abelian topological group, the topology associated (in a standard way) to the continuous convergence structure is the finest of all those which induce the topology of simple convergence on the corresponding equicontinuous subsets. If the starting group is furthermore metrizable (or even almost metrizable), we obtain that such a topology coincides with the compact-open topology. This result constitutes a generalization of the theorem of Banach-Dieudonné, which is well known in the theory of locally convex spaces.

We also characterize completeness, in the class of locally quasi-convex metrizable groups, by means of a property which we have called the quasi-convex compactness property, or briefly $q c p$ (Section 3 ).

2000 Mathematics subject classification: primary 22A05; secondary 46A04, 46A19.

Keywords and phrases: Complete, metrizable group, continuous convergence structure, equicontinuous weak* topology, compact-open topology, Banach-Dieudonné theorem, dual group, locally quasi-convex group.
\end{abstract}

\section{Introduction}

Let $(E, \tau)$ be a topological vector space, $\mathscr{L} E$ the vector space of all continuous linear forms defined on $E$, and $\sigma(\mathscr{L} E, E)$ the topology on $\mathscr{L} E$ of pointwise convergence on the elements of $E$, or weak* topology. Let us denote by $\tau^{f}(\mathscr{L} E, E)$ the topology on $\mathscr{L} E$ finest of all those which coincide with $\sigma(\mathscr{L} E, E)$ on every equicontinuous subset of $\mathscr{L} E$. (The superscript $f$ stands for finest. In the literature it is frequent to call it just $\tau^{f}$.)

Both authors were partially supported by D.G.I.C.Y.T. BFM 2000-0804-C03-01.

(C) 2003 Australian Mathematical Society 1446-7887/03 $\$$ A2.00+0.00 
Many authors have been concerned with the question of when is $\tau^{f}(\mathscr{L} E, E)$ a locally convex topology. The Banach-Dieudonné theorem provides a positive answer if $(E, \tau)$ is a metrizable locally convex space; for it states that, under these circumstances, $\tau^{f}(\mathscr{L} E, E)$ coincides with the topology of uniform convergence on the precompact subsets of $E$.

The topology $\tau^{f}$ was first introduced by Collins, who gave it the name of equicontinuous weak* topology or ew*-topology. He proved that in general it fails to be a locally convex topology, even if the starting space $E$ is locally convex. Komura gave an example where the ew*-topology fails to be a vector topology, [16]. Wheeler generalized the latter seeing that for a topological vector space $E$ with uncountable dimensional dual $\mathscr{L} E$, the ew*-topology corresponding to the weak topology in $E$ is not a vector topology, [22]. Finally, Valdivia produced a device to obtain non-regular ew*-topologies, [21].

The mentioned notions can be defined and studied in the context of topological abelian groups, and this is precisely the topic of this paper. An appropriate framework to develop them is within the theory of convergence spaces, since $\tau^{f}(\mathscr{L} E, E)$ is the topology 'associated' in a standard way to the continuous convergence structure defined directly in $\mathscr{L} E$. Although this is the point of view adopted by Jarchow in his extensive and deep book on locally convex spaces [15], we think our approachfocused only on one problem-is more direct.

In the first section we state a few elementary ideas about convergence theory in order to make the reading of the paper easier. The main result in the second section is a version of the Banach-Dieudonné theorem for abelian topological groups; its proof leans on the fact that the character group of an abelian metrizable group is a k-space. This was proved independently by Chasco and Außenhofer $[9,1]$. In the third section we give a characterization of completeness for locally quasi-convex metrizable groups, which resembles some properties of Banach spaces studied by Mazur and by Krein and Šmulian. The last section deals with some links between topological abelian groups and topological vector spaces, which allow to claim that the results previously obtained are the natural generalizations of the theorems so well known for the class of topological vector spaces.

\section{The continuous convergence structure}

Before defining the continuous convergence structure, we give the notion of a convergence structure in general.

Let $X$ be a set and suppose that to each $x$ in $X$ is associated a collection $\Xi(x)$ of filters on $X$ satisfying:

(i) The ultrafilter $\{A \subset X: x \in A\}$ is in $\Xi(x)$. 
(ii) If $\mathscr{F} \in \Xi(x)$ and $\mathscr{G} \in \Xi(x)$, then the filter $\mathscr{F} \cap \mathscr{G}=\{F \cup G: F \in \mathscr{F}, G \in \mathscr{G}\}$ also belongs to $\Xi(x)$.

(iii) If $\mathscr{F} \in \Xi(x)$ and $\mathscr{G} \supset \mathscr{F}$ then $\mathscr{G} \in \Xi(x)$.

The family $\Xi$ of all filters $\Xi(x)$ for $x$ in $X$ is called a convergence structure for $X$, the pair $(X, \Xi)$ a convergence space and the filters $\mathscr{F}$ in $\Xi(x)$ are called convergent to $x$. We write $\mathscr{F} \rightarrow x$ instead of $\mathscr{F} \in \Xi(x)$ (see $[12,4]$ ).

Every filter gives rise to a net in a standard way and conversely [13]. Thus, by translating the above properties to the equivalent ones for nets, we could introduce a convergence structure by specifying the convergent nets restricted to some axioms (for more details see [5]). The family of all nets on a set cannot be directly considered to form a set. In order to avoid this inconvenience, we will assume that nets are derived from filters and use them freely, since intuition is more on the side of nets.

Every topology on a set $X$ defines a convergence structure; the topology could be recovered from previous knowledge of the convergent filters or nets. For a convergence structure, restricted only to the above axioms (i)-(iii), there is an associated topology; in fact, the 'convergence closed' subsets satisfy the axioms required to be the closed sets of a topology. However this topology has more convergent filters than the a priori' convergent ones. Topological notions, like continuity, compactness, being Hausdorff, regularity, etc. also make sense for convergence spaces. The reader may find a good survey in [4].

The continuous convergence structure is defined on the set of all continuous functions between two convergence spaces, which in particular can be topological spaces, as follows. Let $X, Y$ be convergence spaces. A net of continuous functions $\left\{f_{\alpha}\right\}_{\alpha \in A} \subset C(X, Y)$ is said to converge to $f \in C(X, Y)$ in the continuous convergence structure $\Lambda\left(f_{\alpha} \stackrel{\Lambda}{\rightarrow} f\right)$ if for every net $\left\{x_{\beta}\right\}_{\beta \in B}$ in $X$ convergent to $x$, the combined net $\left\{f_{\alpha}\left(x_{\beta}\right)\right\}_{(\alpha, \beta) \in A \times B}$ converges to $f(x)$ in $Y$. Here the set $A \times B$ is directed by the 'product direction', that is, $(\alpha, \beta)<\left(\alpha^{\prime}, \beta^{\prime}\right)$ if $\alpha<_{A} \alpha^{\prime}$ and $\beta<_{B} \beta^{\prime}$. It can be easily checked that the continuous convergence structure in $C(X, Y)$ is the coarsest convergence structure for which the evaluation mapping $\omega: C(X, Y) \times X \rightarrow Y$, defined by $(f, x) \rightarrow f(x)$, is continuous. It also makes sense for subsets of $C(X, Y)$.

There is a standard way to define the topology associated to a convergence. In particular, we describe the topology $\tau_{\Lambda}$, associated to the continuous convergence structure $\Lambda$, by the statement:

\section{$L \subset C(X, Y)$ is closed in $\tau_{\Lambda}$ if and only if} for every net $\left\{f_{\alpha}\right\} \subset L$ such that $f_{\alpha} \stackrel{\wedge}{\rightarrow} f$ it follows that $f \in L$.

A set satisfying ( $*$ ) is also $\Lambda$-closed for the convergence theory; so, $\Lambda$ and $\tau_{\Lambda}$ give rise to the same closed sets. However, the convergent nets of $\tau_{\Lambda}$ only coincide with the $\Lambda$-convergent nets when $\Lambda$ derives from a topology, which in general is not the case. 
It is easy to check that $f_{\alpha} \stackrel{\Lambda}{\rightarrow} f$ implies $f_{\alpha} \stackrel{\stackrel{\tau}{\Lambda}^{\prime}}{\rightarrow} f$. So it can be said that $\tau_{\Lambda}$ is a topology coarser than $\Lambda$. In fact it is the finest topology among all those coarser than $\Lambda$.

We will be mainly concerned with the case when $X$ is an abelian topological group, and $Y=\pi$ is the multiplicative group of complex numbers modulus one, endowed with the euclidean topology. The homomorphisms from an abelian topological group $G$ into $T$ are called characters; $\Gamma G$ will denote the group of all continuous characters (operation is defined pointwise). The dual group of $G$ in the sense of Pontryagin is $\Gamma G$ endowed with the compact-open topology, $\tau_{\text {co }}$; we call it just $G^{\wedge}$. The symbols $\sigma(G, \Gamma G)$ and $\sigma(\Gamma G, G)$ stand for the topologies on $G$ and on $\Gamma G$ respectively, given by uniform convergence on the elements of the second space in each pair.

The continuous convergence structure defined on $\Gamma G$ gives rise to a convergence group, from now on denoted by $\Gamma_{c} G$ and called the convergence dual of $G$. In fact, it is straightforward to check that addition of elements and taking the inverse in $\Gamma G$ are $\Lambda$-continuous operations. The convergence dual of a topological group $G$ is no longer a topological group, unless $G$ is locally compact.

\section{The Banach-Dieudonné theorem for abelian topological groups}

Let $(G, \tau)$ be an abelian topological group. The finest topology on $\Gamma G$ of all those which induce $\sigma(\Gamma G, G)$ in the equicontinuous subsets of $\Gamma G$ will be called the gew*-topology (g stands for 'group') or $\tau^{f}(\Gamma G, G)$. The result mentioned in the title of this section will be obtained in two steps. First, we see that $\tau^{f}(\Gamma G, G)$ coincides with the topology associated to the continuous convergence structure in $\Gamma G$ (Theorem 2.2). Next, we prove that in the class of almost metrizable groups, it is precisely the compact-open topology (Theorem 2.5 ).

LEMMA 2.1. Let $G$ be a topological group and $\left\{\varphi_{\alpha}, \alpha \in A\right\} \subset \Gamma G$ a net whose range is an equicontinuous subset. Then $\left\{\varphi_{\alpha}\right\}$ converges to some $\varphi \in \Gamma G$ in $\Lambda$ if and only if it converges to $\varphi$ in $\sigma(\Gamma G, G)$.

PROOF. If $\varphi_{\alpha} \stackrel{\wedge}{\rightarrow} \varphi$ it is straightforward, without any conditions, that $\varphi_{\alpha} \stackrel{\sigma(\Gamma G, G)}{\longrightarrow} \varphi$.

Conversely, let $\varphi_{\alpha} \stackrel{\sigma(\Gamma G, G)}{\longrightarrow} \varphi$. Take any net $\left\{x_{\beta}, \beta \in B\right\}$ in $G$ such that $x_{\beta} \rightarrow x$. For any $W$ neighbourhood of 1 in $\mathbb{T}$, we consider another one $W^{\prime}$ such that $W^{\prime} W^{\prime} \subset W$. Based on the equicontinuity condition, we determine $V \in \mathscr{N}_{G}(0)$ with $\varphi_{\alpha}(V) \subset W^{\prime}$, $\forall \alpha \in A$. By the $\sigma(\Gamma G, G)$-convergence of $\left\{\varphi_{\alpha}\right\}$, there exists $\alpha_{0} \in A$ such that $\varphi_{\alpha}(x) \in \varphi(x) W^{\prime}$ for $\alpha \geq \alpha_{0}$. Pick now $\beta_{0} \in B$ such that $x_{\beta} \in x+V, \forall \beta \geq \beta_{0}$. Thus $\varphi_{\alpha}\left(x_{\beta}\right) \in \varphi_{\alpha}(x+V) \subset \varphi_{\alpha}(x) W^{\prime} \subset \varphi(x) W, \forall(\alpha, \beta) \geq\left(\alpha_{0}, \beta_{0}\right)$; in other words, $\varphi_{\alpha}\left(x_{\beta}\right) \rightarrow \varphi(x)$ in $\mathbb{T}$, which implies that $\varphi_{\alpha} \stackrel{\wedge}{\rightarrow} \varphi$. 
The following relationships hold among the indicated convergences in $\Gamma G$

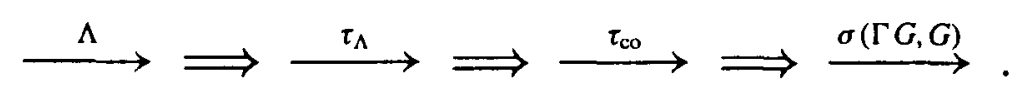

If $M \subset \Gamma G$ is equicontinuous, all of them coincide in $M$ by Lemma 2.1 ; hence, the continuous convergence structure on equicontinuous subsets of $\Gamma G$ is topological.

A distinguished class of equicontinuous subsets of $\Gamma G$ is the one formed by the polars of the family $\mathscr{N}_{G}(0)$ of all neighbourhoods of zero. The polar of a subset $U \subseteq G$ is defined by

$$
U^{\triangleright}:=\left\{\varphi \in \Gamma G: \varphi(U) \subset \mathbb{T}_{+}\right\}
$$

where $\mathbb{T}_{+}:=\{x \in \mathbb{T}: \operatorname{Re}(x) \geq 0\}$, and $\operatorname{Re}$ stands for real part. Evidently $\left\{U^{\triangleright}:\right.$ $\left.U \in \mathscr{N}_{G}(0)\right\}$ is a fundamental family of equicontinuous sets, which means that every equicontinuous subset of $\Gamma G$ is contained in one of those. Note that the sets $U^{\triangleright}$ are closed in $\sigma(\Gamma G, G)$.

We are ready to prove the following:

THEOREM 2.2. Let $G$ be an abelian topological group. The topology associated to the continuous convergence structure $\tau_{\Lambda}$ is the finest of all topologies on $\Gamma G$ which induce $\sigma(\Gamma G, G)$ on all equicontinuous subsets of $\Gamma G$.

PROOF. By Lemma $2.1 \tau_{\Lambda}$ induces $\sigma(\Gamma G, G)$ on all equicontinuous subsets of $\Gamma G$. In order to prove that it is the finest topology with that property, we must check that

$$
C \subset \Gamma G \text { is closed in } \tau_{\Lambda} \text { if and only if }
$$

(**) $C \cap M$ is closed in $M$ with respect to $\tau_{\Lambda \mid M}$, for every equicontinuous $M$.

Since $\tau_{\Lambda}$ is the topology associated to $\Lambda$, the proof can be reduced to show the following: if $C \subset \Gamma G$ satisfies $(* *)$, and $\left\{\varphi_{\alpha}\right\}_{\alpha \in A} \subset C$ is such that $\varphi_{\alpha} \stackrel{\wedge}{\rightarrow} \varphi$, then $\varphi \in C$.

Let us consider the classical net of neighbourhoods of 0 in $G$. Its index set is $B:=\left\{\beta=(x, U) \in G \times \mathscr{N}_{G}(0): x \in U\right\}$, directed under $(x, U) \leq\left(x^{\prime}, U^{\prime}\right)$ if and only if $U^{\prime} \subseteq U$. The net defined by $S_{(x, U)}=x$, converges to 0 , and therefore $\varphi_{\alpha}\left(S_{\beta}\right) \rightarrow 1$. In particular, there exist $\alpha_{0} \in A$ and $\beta_{0}=\left(x_{0}, U_{0}\right) \in B$ such that $\varphi_{\alpha}\left(S_{\beta}\right) \in \mathbb{T}_{+}, \forall(\alpha, \beta) \geq\left(\alpha_{0}, \beta_{0}\right)$. This implies that $\varphi_{\alpha}\left(U_{0}\right) \subset \mathbb{T}_{+}$, and, since $\left\{\varphi_{\alpha}\right\}$ converges pointwise to $\varphi$, also $\varphi\left(U_{0}\right) \subset \mathbb{T}_{+}$. So, for $\alpha \geq \alpha_{0}, \varphi_{\alpha} \in U_{0}^{p}$. Now $U_{0}^{\triangleright}$ is an equicontinuous subset of $\Gamma G$ such that $\varphi_{\alpha} \in C \cap U_{0}^{\triangleright}$, and $\varphi_{\alpha} \rightarrow \varphi \in U_{0}^{\text {D. By the }}$ condition (**) imposed on $C$, we obtain that $\varphi \in C$.

REMARK. In Theorem 2.2 the family of all equicontinuous subsets can be substituted by any fundamental family of equicontinuous sets. 
In [6] it is proved that the topology associated to the continuous convergence structure in a dual group is not necessarily a group topology. After Theorem 2.2, we conclude that the gew*-topology in $\Gamma G$ is not in general a group topology. In order to find conditions under which it is a group topology, as a first approach, we study when it coincides with the compact-open topology. To this end we establish the following lemma.

LEMMA 2.3. Let $G$ be an abelian topological group, and let $\alpha_{G}: G \rightarrow\left(G^{\wedge}\right)^{\wedge}$ be the natural mapping (defined by $\alpha_{G}(g)(\chi)=\chi(g)$ for every $g \in G$ and every $\left.\chi \in G^{\wedge}\right)$. The following assertions hold:

(a) If $M \subset \Gamma G$ is equicontinuous and closed in $\Lambda$, then $M$ is $\Lambda$-compact and conversely.

(b) $\alpha_{G}: G \rightarrow G^{\wedge \wedge}$ is continuous if and only if any $\tau_{\mathrm{co}}$-compact subset of $\Gamma G$ is equicontinuous.

(c) If $\alpha_{G}$ is continuous, then the $\Lambda$-compact and the $\tau_{\mathrm{co}}$-compact subsets of $\Gamma G$ coincide.

Proof. (a) Assume $M \subset \Gamma G$ is equicontinuous. Its closure in $\sigma(\Gamma G, G), \bar{M}^{\sigma}$, is also equicontinuous, and by Ascoli's lemma it must be $\tau_{\text {co }}$-compact. Thus for every net $\left\{\varphi_{\alpha}\right\}_{\alpha \in A} \subset M$ there is a convergent subnet, call it again $\varphi_{\alpha} \stackrel{\tau_{\alpha}}{\rightarrow} \varphi \in \bar{M}^{\sigma}$. By Lemma 2.1, $\varphi_{\alpha} \stackrel{\Lambda}{\rightarrow} \varphi$, and being $M \Lambda$-closed, $\varphi \in M$. Therefore $M$ is $\Lambda$-compact.

Conversely, let $M$ be $\Lambda$-compact. If $M$ were not equicontinuous, for some $W \in$ $\mathscr{N}_{\mathbb{J}}(1)$ and for every $V \in \mathscr{N}_{G}(0)$, there would exist an element $\varphi_{V} \in M$ with $\varphi_{V}(V) \not \subset W$. The net $\left\{\varphi_{V}: V \in \mathscr{N}_{G}(0)\right\}$, where the direction is the usual $\supseteq$-relation. does not have any $\Lambda$-convergent subnet (clearly, every $\varphi_{V}$ can be matched with an element $x_{V} \in V$ such that $\left.\varphi_{V}\left(x_{V}\right) \notin W\right)$. This contradicts the compactness of $M$ in $\Lambda$ (b) This is proved in $[1,(5.10)]$.

(c) Take into account (a) and (b) and the Ascoli lemma stating that every equicontinuous closed subset of $\Gamma G$ is $\tau_{\text {co-compact. }}$ -

PROPOSITION 2.4. Let $G$ be an abelian topological group such that the naturai mapping $\alpha_{G}: G \rightarrow G^{\wedge \wedge}$ is continuous. The following are equivalent:

(a) $\Gamma G$ is a $k$-space with respect to the compact-open topology.

(b) The compact-open topology on $\Gamma G$ is the finest of all those topologies which induce $\sigma(\Gamma G, G)$ on the equicontinuous subsets of $\Gamma G$.

(c) The compact-open topology on $\Gamma G$ coincides with the topology $\tau_{\Lambda}$ associatea to the continuous convergence structure.

ProOF. Observe that by Lemma 2.3 (b) the family of $\tau_{\mathrm{co}}$-compact subsets of $\Gamma G$ coincides with that of equicontinuous $\tau_{\text {co }}$-closed subsets, and those form a fundamenta 
family of equicontinuous subsets. Thus, statement (a) means that the compact-open topology in $\Gamma G$ is the finest of all those which induce $\sigma(\Gamma G, G)$ in the $\tau_{\mathrm{co}}$-compact subsets of $\Gamma G$. So, the equivalence between (a) and (b) is proved. The equivalence between (b) and (c) is obtained from Theorem 2.2.

A natural question now is to find those abelian topological groups $G$ whose dual $G^{\wedge}$ is a k-space. In [9] it is proved that if $G$ is an abelian metrizable group, $G^{\wedge}$ is a k-space. The same assertion holds for the dual of an almost metrizable group [1, (5.20)]. An abelian topological group $G$ is almost metrizable if and only if it contains a compact subgroup $K$ such that $G / K$ is metrizable. The class of almost metrizable groups includes all abelian metrizable groups, all locally compact abelian groups, and more generally, all Čech-complete abelian groups [1, (2.21)]. The above comments together with Proposition 2.4 prove the following:

THEOREM 2.5. If $G$ is an almost metrizable abelian topological group, then the finest of all those topologies which induce $\sigma(\Gamma G, G)$ on the equicontinuous subsets of $\Gamma G$ coincides with the compact-open topology in $\Gamma G$ and therefore it is a group topology.

REMARK. The continuity of $\alpha_{G}$ is implicitly required in Theorem 2.5. In fact, for an almost metrizable group $G, \alpha_{G}$ is already continuous [1, (5.21)]. The weaker assumption of equality between the families of $\tau_{\mathrm{co}}$-compact and $\tau_{\Lambda}$-compact subsets of $\Gamma G$ would also yield the equivalence of (a) and (b) in Proposition 2.4.

It may happen that $\alpha_{G}$ be continuous and $G^{\wedge}$, endowed with the compact-open topology, be not a k-space. In that case $\tau_{\Lambda}$ is the k-extension of $\tau_{c o}$.

For the sake of completeness we reproduce here the example of [6], which, according to Theorem 2.2, proves that the gew*-topology may not be a group topology. Furthermore, it shows that the gew*-topology for the product of two groups is not in general the product of the corresponding gew*-topologies of the factors. In the vector spaces context, it is known that the ew*-topology of a product $E \times F$ is the product of the respective ew*-topologies if $E$ is locally convex and $F$ is finite-dimensional [22, (3.3)].

EXAMPLE 1. Take $G:=\mathbb{R}^{\omega}$, a countable product of real lines with the Tychonoff topology $\tau$ and $G^{\prime}:=\omega \mathbb{R} \subset \mathbb{R}^{\omega}$ its direct sum endowed with $\tau^{\prime}$, the restriction of the box topology of $\mathbb{R}^{\omega}$.

The groups $G$ and $G^{\prime}$ are duals of each other [2, (14.11)]; that is, the following identifications are valid $G^{\wedge} \equiv G^{\prime}$ and $G^{\wedge} \equiv G$.

Since $G$ is metrizable, by Theorem 2.5 the compact-open topology on $G^{\prime}$ coincides with the topology $\tau^{f}(\Gamma G, G)$. On the other hand, since $G$ is a k-space, by 
Proposition 2.4 the compact-open topology coincides with $\tau^{\prime f}\left(\Gamma G^{\prime}, G^{\prime}\right)$. The product group $H:=G \times G^{\prime}$ is selfdual, thus $\alpha_{H}$ is a topological isomorphism, and $\tau^{\prime} \times \tau$ coincides with the corresponding compact-open topology for $H$. In [2, (17.9)], it is proved that $H$ is not a k-space; again by Proposition $2.4, \tau^{\prime} \times \tau$ is not equal to $\left(\tau \times \tau^{\prime}\right)^{f}\left(G^{\prime} \times G, G \times G^{\prime}\right)$.

REMARK. It remains open to characterize those groups $G$ for which the gew* is a group topology in $\Gamma G$. M. J. Chasco has informed us that, for such groups, the corresponding gew*-topology may not coincide with the compact-open topology.

\section{A property of Mazur-type for abelian topological groups}

After [19], we will say that a locally convex space $E$ has the convex compactness property (shortly, $c c p$ ) if the closed circled convex hull of every compact subset $K \subset E$ is again compact. Mazur proved that a Banach space has the ccp; later, Krein and Šmulian proved that a Banach space endowed with its weak topology has the ccp. In this line, it is proved in [19] the following:

'A metrizable locally convex space is complete if and only if it has the ccp'

We shall extend statement (A) to the larger class of locally quasi-convex metrizable groups. We recall first some definitions connected with quasi-convexity; observe that all these concepts, as well as the names used, correspond to analogous notions in locally convex spaces. A subset $M$ of an abelian topological group $G$ is quasi-convex if for every $x \in G \backslash M$ there exists a continuous character $\varphi$ such that $\varphi(M) \subset \mathbb{J}_{+}$and $\varphi(x) \notin \mathbb{J}_{+}$. The quasi-convex hull $Q(H)$ of a subset $H \subset G$ is the intersection of all quasi-convex subsets of $G$ containing $H$. It can be easily checked that it coincides with the bipolar

$$
\left(H^{\triangleright}\right)^{\triangleleft}:=\left\{x \in G: \varphi(x) \in \mathbb{T}_{+}, \forall \varphi \in H^{\triangleright}\right\}
$$

Observe that the quasi-convex hull of a subset of $G$ depends only on $\Gamma G$. If $\nu$ denotes another group topology on $G$ such that $\Gamma(G, v)=\Gamma G$, we will say that $v$ is compatible for the duality $(G, \Gamma G)$ (see [10] for a detailed description of group dualities). The quasi-convex subsets of $G$ are $\sigma(G, \Gamma G)$-closed, therefore closed in the topology of $G$, and also closed in any group topology compatible for the duality $(G, \Gamma G)$.

An abelian Hausdorff topological group $G$ is locally quasi-convex if the quasiconvex neighbourhoods of zero constitute a basis for the zero-neighbourhoods. Further information on locally quasi-convex groups is given in [2]. 
We will say that an abelian topological group $G$ has the quasi-convex compactness property $(q c p)$ if the quasi-convex hull of every compact subset of $G$ is again compact. Let us call a topological abelian group von Neumann complete if its closed precompact subsets are compact. Evidently, any complete group is von Neumann complete. Next, we establish some results related with the qcp.

PROPOSITION 3.1. Let $G$ be a locally quasi-convex topological group. If $G$ is von Neumann complete, or if the natural mapping $\alpha_{G}: G \rightarrow G^{\wedge \wedge}$ is onto, $G$ has the qcp.

PROOF. Let $K \subset G$ be compact. Then $K^{\triangleright}$ is a zero neighbourhood in $G^{\wedge}$, and $K^{\triangleright \triangleright}$ is compact in $G^{\wedge \wedge}$. Being $G$ locally quasi-convex, $\alpha_{G}: G \rightarrow G^{\wedge \wedge}$ is injective and open onto its image [2, (14.3)], that is, $\alpha_{G}^{-1}: \alpha_{G}(G) \rightarrow G$ is continuous. Therefore $\left(K^{\triangleright}\right)^{\triangleleft}=\alpha_{G}^{-1}\left(K^{\triangleright \triangleright} \cap \alpha_{G}(G)\right)$ is precompact in $G$. On the other hand, it is $\sigma(G, \Gamma G)$ closed, and consequently closed in $G$. By the von Neumann completeness of $G$, we have that $\left(K^{\triangleright}\right)^{\triangleleft}$ is compact.

Notice also that, if $\alpha_{G}: G \rightarrow G^{\wedge \wedge}$ is onto, by the above arguments $\alpha_{G}^{-1}$ is a continuous mapping. Thus $\alpha_{G}^{-1}\left(K^{\triangleright \triangleright}\right)=\left(K^{\triangleright}\right)^{\triangleleft}$ is compact for every compact $K \subset G$.

Proposition 3.2. Let $G$ be a locally quasi-convex group. The quasi-convex hull of every precompact subset of $G$ is again precompact.

Proof. Let us call $\tilde{G}$ the completion of $G$, which is a locally quasi-convex group [3]. By Proposition 3.1, $\tilde{G}$ has the qcp. Denote by $M$ a precompact subset of $G$. After identification of $G$ and the corresponding dense subgroup of $\tilde{G}$, we can claim that $Q_{G}(M) \subseteq Q_{\tilde{G}}(M)$ (the subscripts $G, \tilde{G}$ indicate the group in which the quasi-convex hull is to be taken). Now $\tilde{M}$ is compact, and $Q_{\tilde{G}}(\tilde{M})$ is also compact. We obtain that $Q_{G}(M)$ is precompact taking into account that $Q_{G}(M) \subseteq Q_{\tilde{G}}(M)=Q_{\tilde{G}}(\tilde{M})$.

Proposition 3.3. Let $G$ be an abelian topological group and let $\tau_{1}, \tau_{2}$ be locally quasi-convex topologies on $G$, compatible for the duality $(G, \Gamma G)$, and such that $\tau_{1}<\tau_{2}$. If $\left(G, \tau_{1}\right)$ has the qcp, so does $\left(G, \tau_{2}\right)$.

PROOF. Take $K \subset G \tau_{2}$-compact. In particular it is $\tau_{1}$-compact, and by the assumption, $Q(K)$ is also $\tau_{1}$-compact. Thus it is $\tau_{1}$-complete. In order to prove that $\left(Q(K), \tau_{2}\right)$ is also complete, take a $\tau_{2}$-Cauchy net $\left\{x_{\alpha}, \alpha \in A\right\}$. Since $\tau_{1}<\tau_{2}$, it is $\tau_{1}$-Cauchy, and therefore $\tau_{1}$-convergent to some $x \in G$. By [10, (3.9 b))], there exists a zero neighbourhood basis for $\tau_{2}$ formed by $\sigma(G, \Gamma G)$-closed subsets, which consequently are also $\tau_{1}$-closed. Take $V$ symmetric in such a basis, and let $\alpha_{0} \in A$ be such that $x_{\alpha}-x_{\beta} \in V$ for all $\alpha, \beta \geq \alpha_{0}$. Fixing $\beta \geq \alpha_{0}$, and taking into account 
that $V$ is $\tau_{1}$-closed, we have that $x \in x_{\beta}+V$. Since this holds for all $\beta \geq \alpha_{0}$, we obtain that $\left\{x_{\alpha}, \alpha \in A\right\}$ is eventually in $x+V$, and thus it converges to $x$ in $\tau_{2}$.

On the other hand, by Proposition 3.2, $Q(K)$ is precompact in $\tau_{2}$. So $Q(K)$ is $\tau_{2}$-compact.

PROPOSITION 3.4. Let $G$ be an abelian topological group for which the natural mapping $\alpha_{G}$ is continuous. Then $G^{\wedge}$ has the qcp.

ProOF. First we prove that the quasi-convex hull of an equicontinuous subset of $\Gamma G$ is again equicontinuous. Let $M \subset \Gamma G$ be equicontinuous. There exists $V \in \mathscr{N}_{G}(0)$ such that $M \subset V^{\triangleright}$. Now $Q(M)=M^{\triangleright \triangleleft} \subset\left(V^{\triangleright}\right)^{\triangleright \triangleleft}=V^{\triangleright}$, which implies that $Q(M)$ is equicontinuous. Finally, by Lemma $2.3(\mathrm{~b})$, we obtain that $G^{\wedge}$ has the qcp.

COROLLARY 3.5. Every reflexive group $G$ has the qcp.

Taking into account the ideas involved in Section 4 , it is clear that the following theorem is an extension of statement (A).

THEOREM 3.6. A locally quasi-convex metrizable group $G$ is complete if and only if the quasi-convex hull of every compact subset $K \subset G$ is again compact (that is, if $G$ has the $q c p)$.

PROOF. The direct implication holds even without the assumption of metrizability (Proposition 3.1).

The converse implication can be derived from the following facts:

(1) For any abelian metrizable group $G$ there exists a metrizable complete group $\tilde{G}$ which densely contains $G$.

(2) The compact-open topology for the dual of a complete or even of a von Neumann complete, group $H$ coincides with the topology of uniform convergence on the precompact subsets of $H$.

(3) For any dense subgroup $H$ of a metrizable topological group $G$, the dual mapping of the inclusion is a topological isomorphism. Thus $H^{\wedge}$ and $G^{\wedge}$ can be algebraically and topologically identified (See $[9,1]$ ).

(4) If $G$ is a metrizable abelian group and $D \subset G$ a dense subspace, every neighbourhood of zero $V$ in $G^{\wedge}$ contains the polar of an adequate null sequence in $D,[1$, (4.3)].

Take a Cauchy sequence $L:=\left\{x_{n}: n \in \mathbb{N}\right\} \subset G$. Its range $L$ is a precompact set in $\tilde{G}$, thus $L^{\triangleright}$ is a zero-neighbourhood in $\tilde{G}^{\wedge}$. By fact (4), there exists $\left\{a_{n}: n \in \mathbb{N}\right\} \subset G$ with $a_{n} \rightarrow 0$ such that $\left\{a_{n}: n \in \mathbb{N}\right\}^{\triangleright} \subset L^{\triangleright}$. Now $\left\{a_{n}: n \in \mathbb{N}\right\}^{\triangleright \triangleleft} \supset L^{\triangleright \triangleleft} \supset\left\{x_{n}: n \in \mathbb{N}\right\}$. The set $\left\{a_{n}: n \in \mathbb{N}\right\}^{\triangleright \triangleleft}$ is compact by the assumption, and, being metrizable, it is also sequentially compact. This means that the Cauchy sequence $\left\{x_{n}: n \in \mathbb{N}\right\}$ has 
a subsequence convergent to some $x \in\left\{a_{n}: n \in \mathbb{N}\right\}^{\infty 4}$. Therefore, $x_{n} \rightarrow x$ and the group $G$ is complete.

REMARK. If metrizability is dropped, the assertion of Theorem 3.6 does not hold in general. The example given by Komura of a reflexive, non-complete topological vector space $E$, considered as an abelian topological group, would suit as a counterexample. The natural mapping $\alpha_{E}: E \rightarrow E^{\wedge \wedge}$ is onto, therefore, by Proposition 3.1, $E$ has the qcp.

COROLLARY 3.7. The additive group of the rational numbers does not have the qcp.

Observe that a direct proof of the previous statement would entail tedious calculations of quasi-convex hulls. We point out that there are other totally inconnected groups which do have the qcp. For instance, the additive group of integers, being the dual group of $\mathbb{T}$, by Proposition 3.4, has the qcp.

\section{Topological vector spaces as abelian topological groups}

Let $E$ be a real topological vector space. If only addition is considered, $E$ is an abelian topological group. Let us call $\mathscr{L}_{c} E$ the dual space of $E$ endowed with the continuous convergence structure. It is a convergence vector space in the natural sense, and viewed as a group, $\mathscr{L}_{c} E$ is bicontinuously isomorphic to $\Gamma_{c} E$. Let us establish more precisely the parallelism between $\mathscr{L} E$ and $\Gamma E$ :

PROPOSITION 4.1. If $E$ is a topological vector space, the exponential mapping $\rho: \mathscr{L} E \rightarrow \Gamma E$ defined by $\rho(f)=\exp (2 \pi$ if $)$, satisfies the following assertions:

(a) $\rho$ is an algebraic isomorphism.

(b) $\rho: \mathscr{L}_{c} E \rightarrow \Gamma_{c} E$ is a bicontinuous isomorphism.

(c) $\rho:\left(\mathscr{L} E, \tau_{\Lambda}\right) \rightarrow\left(\Gamma E, \tau_{\Lambda}\right)$ is a topological homeomorphism.

(d) $\rho: \mathscr{L}_{\mathrm{co}} E \rightarrow E^{\wedge}$ is a topological isomorphism.

(e) $\rho:(\mathscr{L} E, \sigma(\mathscr{L} E, E)) \rightarrow(\Gamma E, \sigma(\Gamma E, E))$ is a continuous isomorphism, which is not open (unless $\mathscr{L} E=\{0\}$ ).

PROof. Observe that all isomorphisms mentioned are group-isomorphisms.

(a) and (d) are proved in [2, (2.3)].

(b) This is $[8$, Satz 1$]$.

(c) The symbol $\tau_{\Lambda}$ has two different interpretations, but no confusion is likely. The continuity of the natural identities $j: \Gamma_{c} E \rightarrow\left(\Gamma E, \tau_{\Lambda}\right)$ and $k: \mathscr{L}_{c} E \rightarrow\left(\mathscr{L} E, \tau_{\Lambda}\right)$, together with (b) show that the mappings $j \rho: \mathscr{L}_{c} E \rightarrow\left(\Gamma E, \tau_{\Lambda}\right)$ and $\rho^{-1} k: \Gamma_{c} E \rightarrow$ $\left(\mathscr{L} E, \tau_{\Lambda}\right)$ are continuous. Now apply the following general principle whose proof 
is easy: A mapping $f$ from a convergence space $(X, \Xi)$ into a topological space $Y$ is continuous if and only if it is continuous from $X$ with the associated topology $\tau_{\Xi}$ into $Y$.

(e) The continuity is straightforward. If $\mathscr{L} E \neq 0, \rho$ cannot be a topological isomorphism. In the simplest case of $E=\mathbb{R}$, we can identify algebraically $\Gamma \mathbb{R}$ and $\mathscr{L} \mathbb{R}$ with $\mathbb{R}$. However $(\Gamma \mathbb{R}, \sigma(\Gamma \mathbb{R}, \mathbb{R}))$ is a totally bounded subgroup of $\mathbb{T}^{\mathbb{R}}$, while $(\mathscr{L} \mathbb{R}, \sigma(\mathscr{L} \mathbb{R}, \mathbb{R}))$ can be topologically identified with the real line.

It must also be stated that there is a 'good' correspondence between the equicontinuous subsets of $\mathscr{L} E$ and of $\Gamma E$; more precisely, from the next lemma we can conclude that $\rho$ carries a fundamental family of equicontinuous subsets of $\mathscr{L} E$ into a fundamental family of equicontinuous subsets of $\Gamma E$.

In order to distinguish 'polar sets' in topological vector spaces from polars in topological groups, for $M \subset E$ let us denote by

$$
M^{\circ}:=\{f \in \mathscr{L} E:|f(m)| \leq 1, \forall m \in M\}
$$

and by

$$
{ }^{\circ}\left(M^{\circ}\right):=\left\{x \in E:|f(x)| \leq 1, \forall f \in M^{\circ}\right\}
$$

LEMMA 4.2. Let $U$ be a circled neighbourhood of zero in $E$. Then $\rho\left((4 U)^{\circ}\right)=U^{\triangleright}$. Furthermore, ${ }^{\circ}\left(U^{\circ}\right)=\left(U^{\circ}\right)^{\circ}$.

PrOOF. This is Proposition 1.11 of [10].

Clearly by Lemma 4.1 (d), $\rho$ considered as a mapping from $(4 U)^{\circ}$ into $U^{\triangleright}$ is a homeomorphism if both of them are endowed with the corresponding compact-open topology, which in turn coincides with $\sigma(\mathscr{L} E, E)$ and with $\sigma(\Gamma E, E)$ respectively.

By the above comments, $\rho$ is a homeomorphism from $\left(\mathscr{L} E, \tau^{f}(\mathscr{L} E, E)\right)$ into $\left(\Gamma E, \tau^{f}(\Gamma E, E)\right)$, and the following claims are the transcriptions of Theorem 2.2 and Theorem 2.5.

COROLLARY 4.3. Let E be a topological vector space. The topology associated to the continuous convergence structure on $\mathscr{L} E$ is the finest of all those which induce $\sigma(\mathscr{L} E, E)$ on the equicontinuous subsets of $\mathscr{L} E$.

COROLLARY 4.4. Let $E$ be a metrizable topological vector space. The compactopen topology on $\mathscr{L} E$ is the finest of all those which induce $\sigma(\mathscr{L} E, E)$ on the equicontinuous subsets of $\mathscr{L} E$.

Corollary 4.4 is essentially the Banach-Dieudonné theorem. However in the vast literature existing, as far as we know, it is only stated for locally convex spaces. 
In the previous section we defined the qcp, a property for groups 'parallel' to the convex compactness property (ccp) for locally convex spaces. We check now that both notions coincide in the realm of locally convex spaces.

Let $E$ be a topological vector space and $A \subset E$ a subset. Denote by $\mathrm{e}(A)$ and co(e $(A))$ the circled and the convex circled hull of $A$, respectively.

PROPOSITION 4.5. A locally convex space $E$ has the qcp if and only if $E$ has the ccp.

PROOF. If $K$ is a compact subset of $E$, it is easy to see that $\mathrm{e}(K)$ is also compact. By the bipolar theorem, $\overline{\operatorname{co}(\mathrm{e}(K))}={ }^{\circ}\left(K^{\circ}\right)={ }^{\circ}\left((\mathrm{e}(K))^{\circ}\right)$, and, being $\mathrm{e}(K)$ circled, ${ }^{\circ}\left((\mathrm{e}(K))^{\circ}\right)=\left((\mathrm{e}(K))^{\triangleright}\right)^{\triangleleft}=Q(\mathrm{e}(K))$.

If $E$ has the qcp, then $\overline{\operatorname{co}(\mathrm{e}(K))}=Q(\mathrm{e}(K))$ is compact.

Conversely, if $E$ has the ccp, the quasi-convex hull $Q(K) \subseteq Q(\mathrm{e}(K))=\overline{\operatorname{co}(\mathrm{e}(K))}$ is a closed subset of a compact set; thus $Q(K)$ is compact.

COROLLARY 4.6. A complete locally convex space E equipped with the Bohr topology $\sigma\left(E, E^{\wedge}\right)$ has the qcp.

PROOF. According to Krein theorem, the space $E$ endowed with its weak topology $\sigma\left(E, E^{*}\right)$ has the ccp. By Proposition 4.5 , it also has the qcp. The assertion follows now from the fact that, the $\sigma\left(E, E^{\wedge}\right)$-compact subsets of $E$ are exactly the $\sigma\left(E, E^{*}\right)$ compact subsets [10].

REMARK. A subtle difference between locally convex spaces and locally quasiconvex groups: For a locally convex space $E, \alpha_{E}$ is onto if and only if $E$ has the ccp or, equivalently, if it has the qcp. However the group of all almost everywhere integer valued functions $G:=L_{\mathbb{Z}}^{2}([0,1])$, with the topology as a subspace of $L^{2}([0,1])$, is a complete metrizable locally quasi-convex group. By Proposition 3.1, $G$ has the qcp; however $\alpha_{G}$ is not onto [1, (11.14)]. In this respect, it would be interesting to obtain the result of the last corollary for locally quasi-convex groups.

REMARK. Although the definition of the associated topology $\tau_{\Lambda}$ is rather misty, a geometric description of a zero-neighbourhood basis for the dual of a separable Hilbert space is given in [18]. Since the original paper may be difficult to read, we reproduce here the mentioned basis.

Denote by $\mathscr{H}$ a separable Hilbert space, by $B_{r}^{(p)}$ a ball of radius $r$ in a subspace $L^{(p)}$ of codimension $p$ and, for $\varepsilon>0, N_{\varepsilon}(A)$ stands for the $\varepsilon$-neighbourhood in $\mathscr{H}$ of a subset $A \subset \mathscr{H}$. The sets of the form

$$
B_{r_{0}}^{(0)} \cup \bigcup_{i=1}^{\infty} N_{\varepsilon_{i}}\left(B_{r_{i}}^{\left(p_{i}\right)}\right),
$$


$\left(p_{i}\right)$ and $\left(r_{i}\right)$ being increasing sequences of natural and real numbers respectively, such that $r_{i} \rightarrow \infty$ and $\varepsilon_{i}>0$, for every $i$, constitute a neighbourhood basis of zero for the topology $\tau_{\Lambda}$ in $\mathscr{H}$.

\section{Acknowledgements}

The authors are grateful to M. J. Chasco and to V. Tarieladze, for highly instructive conversations. Also to the referee, who gave us the opportunity to improve the first version of the paper.

NOTE. After sending the final version of the present paper, we have learned that S. Hernández had also obtained the result of Theorem 3.6, published in [14]. Nevertheless, our approach is different.

\section{References}

[1] L. Außenhofer, 'Contributions to the duality theory of abelian topological groups and to the theory of nuclear groups', Dissertationes Math. 384 (1999).

[2] W. Banaszczyk, Additive subgroups of topological vector spaces, Lecture Notes in Math. 1466 (Springer, Berlin, 1991).

[3] W. Banaszczyk and E. Martín-Peinador, 'Weakly pseudocompact subsets of nuclear groups', $J$. Pure Appl. Algebra 138 (1999), 99-106.

[4] E. Binz, Continuous Convergence in $C(X)$, Lecture Notes in Math. 469 (Springer, Heidelberg, 1975).

[5] M. Bruguera, Grupos topológicos y grupos de convergencia: estudio de la dualidad de Pontryagin (Ph.D. Thesis, University of Barcelona, 1999).

[6] M. Bruguera and M. J. Chasco, 'Strong reflexivity of abelian groups', Czechoslovak Math. J. 51 (2001), 213-224.

[7] M. Bruguera, M. J. Chasco, E. Martín-Peinador and V. Tarieladze, 'Completeness properties of locally quasi-convex groups', Topology Appl. 111 (2001), 81-93.

[8] H. P. Butzmann, 'Pontrjagin-Dualität für topologische Vektorräume', Arch. Math. 28 (1977), 632-637.

[9] M. J. Chasco, 'Pontryagin duality for metrizable groups', Arch. Math. 70 (1998), 22-28.

[10] M. J. Chasco, E. Martín-Peinador and V. Tarieladze, 'On Mackey topology for groups', Studia Math. 132 (1999), 257-284.

[11] H. S. Collins, 'Completeness and compactness in linear topological spaces', Trans. Amer. Math. Soc. 79 (1955), 256-280.

[12] H. R. Fischer, 'Limesräume', Math. Ann. 137 (1959), 269-303.

[13] M. García, J. Margalef, C. Olano de Lorenzo, E. Outerelo and J. L. Pinilla, Topología I (Ed. Alhambra, Madrid, 1975).

[14] S. Hernández, 'Pontryagin duality for topological abelian groups', Math. Z. 238 (2001), 493-503.

[15] H. Jarchow, Locally convex spaces (B. G. Teubner, Stuttgart, 1981). 
[16] Y. Komura, 'Some examples on linear topological spaces', Math. Ann. 153 (1964), 150-162.

[17] G. Köthe, Topological vector spaces I, Grundlehren Math. Wiss. 159 (Springer, New York, 1969).

[18] E. Martín-Peinador, 'Construcción de la topología de la convergencia débil en el espacio de Hilbert', Rev. Mat. Hispano-Americana, $4^{a}$ Serie 34 (1974), 221-229.

[19] E. G. Ostling and A. Wilansky, 'Locally convex topologies and the convex compactness property', Proc. Cambridge Philos. Soc. 74 (1974), 45-50.

[20] H. H. Schaefer, Topological vector spaces, Graduate Texts in Math. 3 (Springer, Heidelberg, 1970).

[21] M. Valdivia, 'On certain topologies on a vector space', Manuscripta Math. 14 (1974), 241-247.

[22] R. F. Wheeler, 'The equicontinuous weak* topology and semi-reflexivity', Studia Math. 41 (1972), 243-256.

Dept. de Matemática Aplicada I

Universidad Politécnica de Cataluña

Spain

e-mail: m.montserrat.bruguera@upc.es
Dept. de Geometría y Topología Universidad Complutense de Madrid Spain e-mail: peinador@mat.ucm.es 